\title{
Pyk2 controls filamentous actin formation in human glomerular mesangial cells via modulation of profilin expression
}

This article was published in the following Dove Press journal:

Cell Health and Cytoskeleton

II June 2009

Number of times this article has been viewed

\author{
Victoriya A Rufanova' \\ Anna Alexanian' \\ Tetsuro Wakatsuki ${ }^{2,3}$ \\ Andrey Sorokin' \\ 'Department of Medicine, Division \\ of Nephrology, Kidney Disease Center \\ Milwaukee, WI, USA; '2Department \\ of Physiology, ${ }^{3}$ Bioengineering \\ and Biotechnology Center, Medical \\ College of Wisconsin, Milwaukee, \\ WI, USA
}

\begin{abstract}
In glomerular mesangial cell (GMC), important regulators of glomerular filtration, adenovirus-mediated overexpression of calcium regulated nonkinase (CRNK), a dominant interfering calcium-regulated nonreceptor proline-rich tyrosine kinase 2 (Pyk2) construct, inhibited Pyk2 activity and caused enhanced RhoA activity, enriched cortical actin formation at time of cell replating, and reduction of spreading. We aimed to further explore Pyk2 regulation of the actin dynamic during cell spreading as a vital characteristic of GMC function. GMC were infected with adenovirus encoding CRNK or green fluorescent protein (GFP) as a control and 48 hours after infection cells were harvested and either re-plated or left in suspension for one hour. De novo adhesion to substrate was significantly decreased after Pyk2 activity inhibition and was further diminished after treatment with Rho-associated kinase inhibitor. Inhibition of Pyk2 was associated with increased filamentous actin formation and a corresponding decrease in globular to filamentous actin ratio during cell spreading. Phosphorylation and expression of cofilin, a RhoA-regulated filamentous actin destabilizing factor, were similar in CRNK-expressing and control GMC. Expression of profilin, an activator of actin polymerization, was enhanced, whereas phosphorylation of Pyk2 and p130Cas was decreased. Our data suggest that Pyk2 signaling controls the filamentous actin formation during cell spreading via upregulation of profilin expression.
\end{abstract}

Keywords: Pyk2, profilin, cell spreading, adhesion, glomerular mesangial cells, p130Cas, actin dynamic, ROCK inhibition

\section{Introduction}

Calcium-regulated nonreceptor proline-rich tyrosine kinase 2, or Pyk2 (also known as RAFTK, FAK2, CAK $\beta$, and CADTK), is a cytoplasmic protein tyrosine kinase that, together with the focal adhesion kinase (FAK), belongs to a unique subfamily of tyrosine kinases. ${ }^{1}$ Pyk 2 can play a crucial role in the cytoskeleton rearrangements involved in migration, contraction and adhesion, exhibiting tissue-specific function. ${ }^{2-6}$ Previously we demonstrated that Pyk2 is expressed and can be activated in glomerular mesangial cell (GMC), ${ }^{7}$ which are important regulators of glomerular filtration in normal physiological conditions as well as in renal pathologies (diabetic nephropathy, hypertensive glomerulopathy, and glomerulonephritis). ${ }^{8}$ We described that decrease in basal endogenous Pyk2 activity is associated with 1) enhanced GTP-loading of RhoA, a member of the Ras superfamily of small GTPases, in quiescent cultured GMC; 2) decreased cell adhesion and spreading; 3) increased cortical filamentous actin formation during cell spreading as seen by filamentous actin visualization. ${ }^{9}$ Our findings suggest that Pyk 2 can be involved into GMC cytoskeleton rearrangements, mediating its effects in parallel or/and
Correspondence: Andrey Sorokin Medical College of Wisconsin, Department of Medicine/Kidney Disease Center, 870 I Watertown Plank Rd, Milwaukee, WI 53226, USA

Tel + I $4 \mid 44564438$

Fax + I 414456 65I5

Email sorokin@mcw.edu 
in synergy with Rho-associated kinase (ROCK). Indeed, many actin-anchored structures such as focal adhesions are active sites of intracellular signaling. ${ }^{10}$ Integrin receptor clustering in focal adhesions is critical for the activation of protein tyrosine kinases of Pyk2/FAK and Src families and members of the Rho family of small GTPases. ${ }^{11}$

The actin cytoskeleton and a network of signaling pathways originating from cell surface receptors cooperate to promote an appropriate response of the cell. During morphological changes, the actin cytoskeleton responds to signals by providing a structural frame, but it also organizes signaling pathways and it may perform signaling functions by itself. Both the assembly and the disassembly of actin filaments is regulated by RhoA, ${ }^{12}$ which has two major effectors: ROCK and mDia (mammalian homolog of Drosophila diaphanous). The mDia is a formin molecule that catalyzes actin nucleation and polymerizes long actin filaments. ${ }^{13}$ Profilin mediates incorporation of ATP-bound globular actin into the barbed $(+)$ end of the filaments. Cofilin severs and dissociates the filamentous actin at their pointed (-) end. RhoA activates profilin via mDia, while cofilin is inactivated by phosphorylation via LIM kinase, which is under the control of ROCK. Polymerizing actins are ATP-bound but as they get older in filaments ATP get hydrolyzed to ADP. ${ }^{14-16}$

Originally described as an actin-binding protein, profilin has now been shown to interact with more than a dozen proteins in mammalian cells. Some of the more recently intriguing interactions are within neurons involving a neuronal profilin family member. ${ }^{17}$ Recently profilin has been identified as direct target of ROCK1, inhibiting its globular actin polymerizing activity. ${ }^{18}$ Y-27632 blocks the ROCKmediated phosphorylation of profilin Ser-137 in HEK293 cells and primary neurons, which maintains profilin in an active state. Profilin is now described as a regulator of various cellular processes such as cytoskeletal dynamics, membrane trafficking, and nuclear transport. Profilin is a necessary element in key steps of neuronal differentiation and synaptic plasticity, and embodies properties postulated for a synaptic tag. Some data indicate that profilin might exhibit cell-specific function, suggesting that in kidney cells regulation and activity of profilin can exhibit different pattern compared to our understanding gained in other systems.

The actin cytoskeleton has important functions in various biological processes in all eukaryotic cells including cell motility, migration and adhesion. ${ }^{19}$ The regulation of the cytoskeleton has therefore a central role in normal physiology as well as in diseases such as diabetes, hypertension, and renal inflammation. Currently the link between Pyk2 and cellular actin dynamic in GMC remains largely unknown. In this study we aimed to investigate relationship between Pyk2 activity and key regulators of actin de- and polymerization, cofilin and profilin, respectively, during cell spreading as an important function of mesangial cells.

\section{Materials and methods Materials}

The enhanced chemiluminescence detection system kit was supplied by Amersham Pharmacia Biotech (Baie d'Urfé, QC, Canada). The BCA protein assay kit was from Pierce (Rockford, IL). All other reagents, unless different indicated, were from Sigma (St. Louis, MO).

\section{Antibodies}

For the Western blotting analysis rabbit polyclonal antip130Cas, anti-total Pyk2, and mouse monoclonal anti-FLAG antibodies were obtained from Santa Cruz Biotechnology (Santa Cruz, CA). Mouse monoclonal anti-actin antibody was from ICN Biochemicals (Costa Mesa, CA). Polyclonal phosphorylation state-specific anti-Pyk2 Y402 antibody was from Biosource International (Camarillo, CA). Rabbit anti-Cofilin 1, phospho (Ser3) polyclonal antibody was from ECM Bioscience (Versailles, KY). Mouse monoclonal anti-profilin antibody was from BD Transduction Laboratories (Lexington, KY). Rabbit polyclonal phosphorylation state-specific anti-p130Cas Y165 was from Cell Signaling Technology (Beverly, MA). The horseradish peroxidaseconjugated goat anti-mouse and goat anti-rabbit immunoglobulins were bought from BioRad (Hercules, CA).

\section{Cell culture and adenovirus-mediated gene transfer}

All materials for cell culturing were purchased from Invitrogen (Carlsbad, CA). Previously characterized SV40-transformed human GMC $^{20}$ were cultured in RPMI 1640 supplemented with 10\% fetal bovine serum, $10 \mathrm{mM}$ HEPES, $2 \mathrm{mM}$ glutamine, penicillin (100 units/ml), and streptomycin $(100 \mu \mathrm{g} / \mathrm{ml})$ in a $37{ }^{\circ} \mathrm{C}$ humidified incubator with $5 \% \mathrm{CO}_{2}$. The recombinant adenoviral vector (Ad) encoding the carboxyl terminus of Pyk2 termed CRNK for calcium-dependent tyrosine kinase-related nonkinase (AdCRNK) and control viruses (AdGFP) were constructed and used exactly as described previously. ${ }^{21}$

\section{Cell spreading, lysis, and immunoblot analysis}

AdGFP-, and AdCRNK-infected human GMC were allowing to spread or were left in suspension on ice in serum-free 
media as described previously. ${ }^{9}$ In each experimental condition the cells were lysed, and then subjected to Western blotting analysis as described previously. ${ }^{9,21}$

\section{Adhesion assays}

Cell adhesion in AdGFP- and AdCRNK-infected human GMC were analyzed exactly as described previously ${ }^{9}$ except that serum-free RPMI with or without ROCK inhibitor Y27632 at the final concentration $10 \mu \mathrm{M}$, or with solvent was added into each plate prior to addition of the suspended cells. After one hour incubation at $37^{\circ} \mathrm{C}$, cell adhesion was stopped by removing the medium, washing twice with phosphatebuffered saline (PBS), and incubating with $70 \%$ ethanol for 20 minutes. Cells were stained with crystal violet, washed four times with PBS, and the dye was extracted with $0.1 \mathrm{M}$ sodium citrate for $30 \mathrm{~min}$. Optical density was measured at $595 \mathrm{~nm}$ in an automatic plate reader (Synergy-HT; BioTek Instruments, Inc., Winooski, VT). After background subtraction data were present as an average of three experiments.

\section{$\mathrm{G} / \mathrm{F}$ actin assay}

AdGFP- and AdCRNK-infected GMC were detached by trypsinization, and re-suspended in serum-free RPMI. The cell concentration was adjusted to $180,000 \mathrm{cells} / \mathrm{ml}$ for re-plating in $35 \mathrm{~mm}$ tissue culture dishes. Serum-free RPMI $(1 \mathrm{ml})$ was poured into each culture dish prior to addition of the suspended cells. After incubation at $37^{\circ} \mathrm{C}$ for one hour, medium was removed and lysis buffer was added to each condition plate. Filamentous actin (F-actin) and free globular-actin (G-actin) content in GMC were measured using an assay kit obtained from Cytoskeleton Inc. (Denver, CO). Briefly, upon exposure to various stimuli and/or inhibitors, GMC were homogenized in cell lysis and F-actin stabilization buffer $(50 \mathrm{mmol} / \mathrm{L}$ PIPES, $50 \mathrm{mmol} / \mathrm{L} \mathrm{NaCl}, 5 \mathrm{mmol} / \mathrm{L}$ $\mathrm{MgCl}_{2}, 5 \mathrm{mmol} / \mathrm{L}$ EGTA, 5\% (v/v) lyceral, 0.1\% (v/v) Nonidet P-40, 0.1\% (v/v) Triton X-100, 0.1\% (v/v) Tween $20,0.1 \%(\mathrm{v} / \mathrm{v}) 2-\mathrm{mercaptoethanol}$, and $0.001 \%(\mathrm{v} / \mathrm{v})$ antifoam and a protease inhibitor cocktail followed by centrifugation for one hour at $100000 \mathrm{~g}$ to separate the F-actin from $\mathrm{G}$-actin pool. Supernatants of the protein extracts were collected after centrifugation. The pellets were resuspended in ice-cold $d \mathrm{H}_{2} \mathrm{O}$ plus $1 \mu \mathrm{M}$ cytochalasin $\mathrm{D}$ and then incubated on ice for one hour to dissociate F-actin. The resuspended pellets were gently mixed every $15 \mathrm{~min}$. Equal amounts of both the supernatant (G-actin) and the resuspended pellet (F-actin) were subjected to analysis of immunoblot with the use of an actin antibody (Cytoskeleton Inc.). Western blotting data were scanned and subjected to densitometry analysis.
In each experiment final results are presented as $\mathrm{G} / \mathrm{F}$ actin ratio relative to GFP-expressing control.

\section{Statistics}

Data are reported as means \pm SEM unless otherwise indicated. For Western blotting densitometry statistical analysis was performed by one- or two-ways analysis of variance (ANOVA), followed by a Duncan post hoc test. Two-way ANOVA analysis followed by Tukey test was done to estimate differences between the groups in adhesion experiments. The differences between means were considered significant at $p<0.05$.

\section{Results}

\section{Simultaneous inhibition of endogenous Pyk2 and ROCK activities reduces GMC adhesion}

Since Pyk2 was shown to be involved in formation and maturation of focal adhesions, we studied GMC adhesion and spreading as indicators of functional phenotype that could be linked to Pyk2 activity in numerous cell types. ${ }^{22}$ First, we checked GMC adhesion in the presence and absence of ROCK inhibitor, because RhoA activation was described to be important for cell-matrix adhesion site formation. Fibronectin (FN) was chosen as a substrate because it was shown 1) to stimulate Pyk2 activity via $\beta 1$ integrins, ${ }^{23} 2$ ) to be secreted by GCM for regulating its function; ${ }^{24} 3$ ) to be important pathological factor in several renal diseases. ${ }^{25}$ AdGFP infected GMC demonstrated no significant change in the number of cells bound to FN after ROCK inhibition (Figure 1A, white and greyon-white bars). After on hour incubation CRNK expressing GMC attachment to FN was significantly reduced by about $30 \%$ compared to GFP control after one hour incubation (Figure 1A, grey-on-white). Addition of ROCK inhibitor to AdCRNK infected GMC markedly decreased cell attachment even further by about $35 \%$ compared to AdCRNK sample (Figure 1A, dark grey and dark-grey-on-light-grey bars) and by about $50 \%$ compared to AdGFP sample (Figure 1A, greyon-white and dark-grey-on-light-grey bars).

Next we determined whether phosphorylation status of endogenous Pyk 2 is associated with $1 \mathrm{~h}$ of spreading on regular cell culture treated plastic with and without ROCK inhibition (Figure 1B). Consistent with adhesion data (Figure 1A) Pyk2 activation, which was measured by Western blotting with anti-phosphoY402 Pyk2 antibody was reduced after ROCK application in both GMC populations. CRNK overexpression led to a decrease in endogenous Pyk2 activation when 
compared to GFP control (Figure 1B). Equal protein loading was confirmed by immunoblotting of cell lysates with antiactin antibodies.

\section{Inhibition of endogenous Pyk2 activation by CRNK expression led to increased F-actin formation during cell spreading}

Cell spreading is an unique process actively engaging not only integrins, but also actin cytoskeleton transformations. ${ }^{26}$ Previously we described that endogenous Pyk2 inhibition by CRNK was accompanied by enriched peripheral cortical F-actin staining compare to GFP control with diffuse F-actin representation. ${ }^{9}$ In the current study we quantified cellular filamentous actin content by measuring G/F actin ratio. As illustrated on Figure $1 \mathrm{C}$, at one hour after replating CRNK expressing cells had significantly smaller (about 50\% reduction; $\mathrm{p}<0.05, \mathrm{n}=4) \mathrm{G} / \mathrm{F}$ actin ratio compared to GFP expressing GMC. Decrease in G/F ratio reflects relative increase in filamentous actin amount. Similar increase in detectable F-actin amount after CRNK overexpression was observed after cytochalasin D pretreatment of spreading cells. Pretreatment with phalloidin, which suppose to stabilize formed filamentous actin structures, did not reveal statistically significant difference between cell populations.

\section{Inhibition of endogenous Pyk2 phosphorylation associated with increased profilin expression}

We analyzed two key regulators of filamentous actin assembly and disassembly (profilin and cofilin) with and without endogenous Pyk2 activity inhibition. Initially we hypothesized that filamentous actin accumulation in CRNK-expressing cells might be due to increased cofilin Ser-3 phosphorylation, inactivating severing activity of cofilin. Thus, we used anti-cofilin, phosphorylated on Ser-3, antibody to compare GFP- and CRNK-expressing GMC left in suspension or replated for $1 \mathrm{~h}$. Representative results of this experiment are shown on Figure 2A. Cofilin phosphorylation was increased by about $35 \%$ after plating GMC on culture dish compare to suspension. However, we did not observe any difference between GFP- and CRNK-expressing cells, as measured by relative amount of phosphorylated cofilin to actin, acquired from densitometry of Western blotting data (Figure 2A).

Then we analyzed profilin expression as another possible reason for increased filamentous actin formation in substrate attached GMC after Pyk2 inhibition. In accordance with our previously published results, ${ }^{9}$ CRNK overexpression caused significant decrease in phosphorylated tyrosin 402 of Pyk 2 and tyrosin 165 of p130Cas substrate binding domain (Figure 2B, first and second panels from the top). In contrast, the level of endogenous profilin protein, as evaluated by Western blot analysis of cell lysates, was significantly $(\mathrm{p}<0.05, \mathrm{n}=4)$ increased almost twice in cells infected with AdCRNK, when compared to cells treated with control virus (Figure 2B, C). Results of representative experiment are shown in Figure 2B. Equal protein loading was confirmed by immunoblotting of cell lysates with anti-actin antibodies (Figure 2, fourth panel). Summary of four independent experiments analyzing profilin expression normalized by actin presented on Figure $2 \mathrm{C}$.

\section{Discussion}

In the present study we report that in human GMC de novo adhesion to substrate was significantly decreased after inhibition of Pyk2 activity and was further diminished after treatment with ROCK inhibitor, suggesting cross talk between Pyk2 and RhoA signaling during GMC adhesion. Interestingly, there was no difference in GMC adhesion between AdGFP and AdGFP+Y cell populations (Figure 1A). GMC adhesion data correlated with endogenous Pyk2 phosphorylation in the same experimental conditions: 1) one hour attachment, 2) with and without ROCK inhibitor added, and 3) with and without CRNK overexpression, which inhibits endogenous Pyk2 activation (Figure 1B). These data suggest that there is a threshold level of Pyk2 activity, important for support of cell adhesion. ROCK inhibition can decrease Pyk2 activity, but such decrease was not enough to detect significant change in cell adhesion. In AdGFP cells, even after addition of ROCK inhibitor, Pyk2 phosphorylation was still higher compared to both AdCRNK groups with and without Y27632. CRNK overexpression reduced Pyk2 phosphorylations to greater extend when compared to cells treated with ROCK inhibiter, significantly affecting adhesion. Simultaneous application of both inhibitors had synergic effect on cell adhesion and Pyk2 phosphorylation, highlighting key role of Pyk2 signaling during GMC adhesion. We have not detected any changes in cofilin phosphorylation between AdGFP and AdCRNK GMC. RhoA-dependent cofilin phosphorylation was linked to cell spreading, but not adhesion in different cell types. ${ }^{27,28}$.

Hypothetical interactions between Pyk2 and RhoA signaling pathways, resulting in changes in profilin expression, reflected in Figure 3. Heterodimeric integrin receptors could potentially activate Rho via signals originating from cytoplasmic domain of beta-integrin subunit at focal adhesions, and thus the mechanisms activating the Rho and Pyk2 pathways would partially overlap. Pyk2 was found co-localized with 
A)

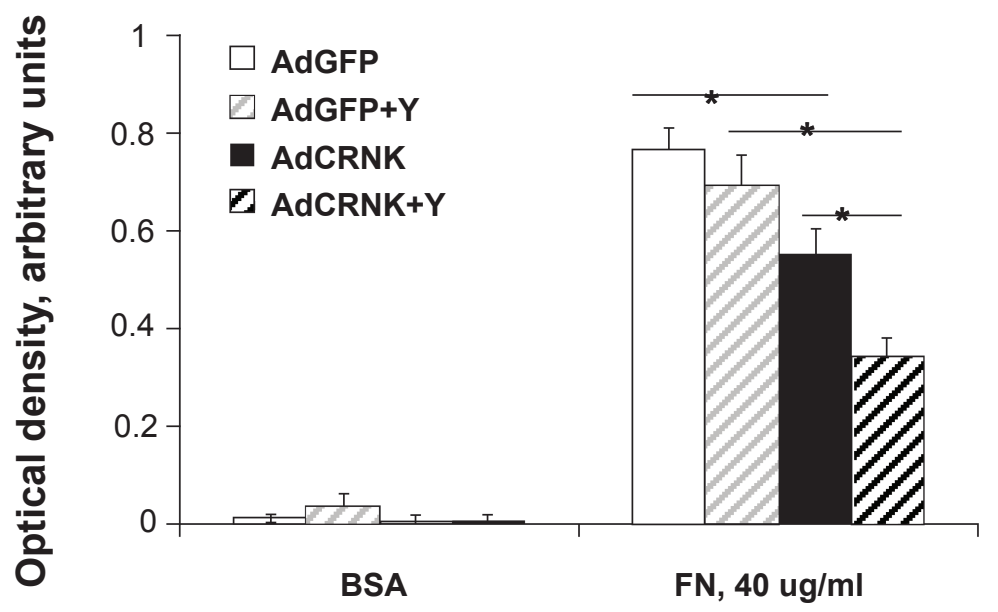

B)

\begin{tabular}{|c|c|c|c|c|}
\hline $\begin{array}{r}\text { Y27632 } \\
\text { Overexpression }\end{array}$ & $\overline{\mathbf{G}}$ & $\bar{c}$ & $\stackrel{+}{\mathbf{G}}$ & $\stackrel{+}{\mathrm{C}}$ \\
\hline pPyk2 & $E$ & 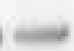 & $\Rightarrow$ & -4 \\
\hline Actin & & & & \\
\hline
\end{tabular}

C)

$1 \mathrm{~h}$ spreading

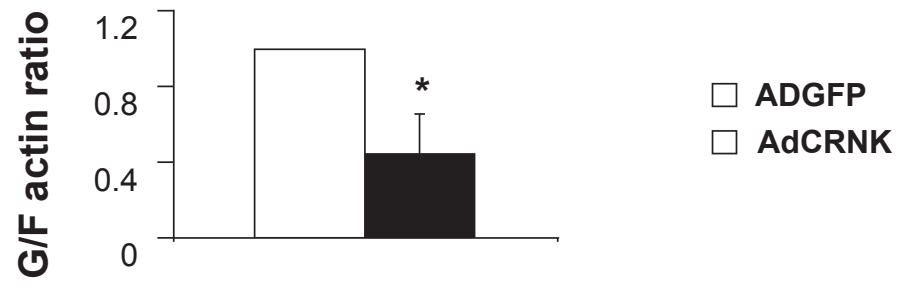

\section{+ Cytochalasin D}

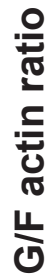

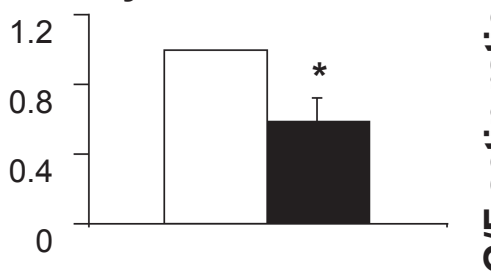

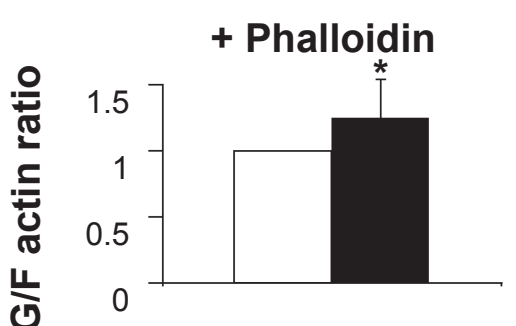

Figure I Pyk2 activity in GMC adhesion. A) Glomerular mesangial cell adhesion depends on endogenous Pyk2 and Rho-associated kinase (ROCK) activities. Graph is a summary of three independent experiments analyzing GMC adhesion (see Methods for details) to fibronectin (FN) or bovine serum albumin (BSA) as a negative control in the presence or the absence of ROCK inhibitor Y27632 (I0 $\mu$ M) one hour after plating. B) Pyk2 phosphorylation decreased after ROCK inhibitor application in GMC. GFP- (marked as "G" on corresponding lanes) or CRNK-expressing (marked as "C" on corresponding lanes) GMC were allowed to spread for one hour in presence or absence of Y27632, washed three times, lysed and subjected to Western blotting analysis with anti-phosphorylated tyrosin 402 of Pyk2 (top panel) and anti-actin (bottom panel) antibody. C) Inhibition of endogenous Pyk2 phosphorylation associated with increase of relative to globular filamentous actin formation. Quiescent AdGFP- and AdCRNK-infected human GMC were allowed to spread for one hour, then washed with PBS three times and lysed. Total cell lysates were subjected to G/F actin assay as described in Methods. Graphical results of four independent experiments presented. $\mathrm{P}<0.05$ was considered significant and marked as * on the graph.

Abbreviations: CRNK, calcium regulated nonkinase; GFP, green fluorescent protein; GMC, glomerular mesangial cell; Pyk2, proline-rich tyrosine kinase 2. 

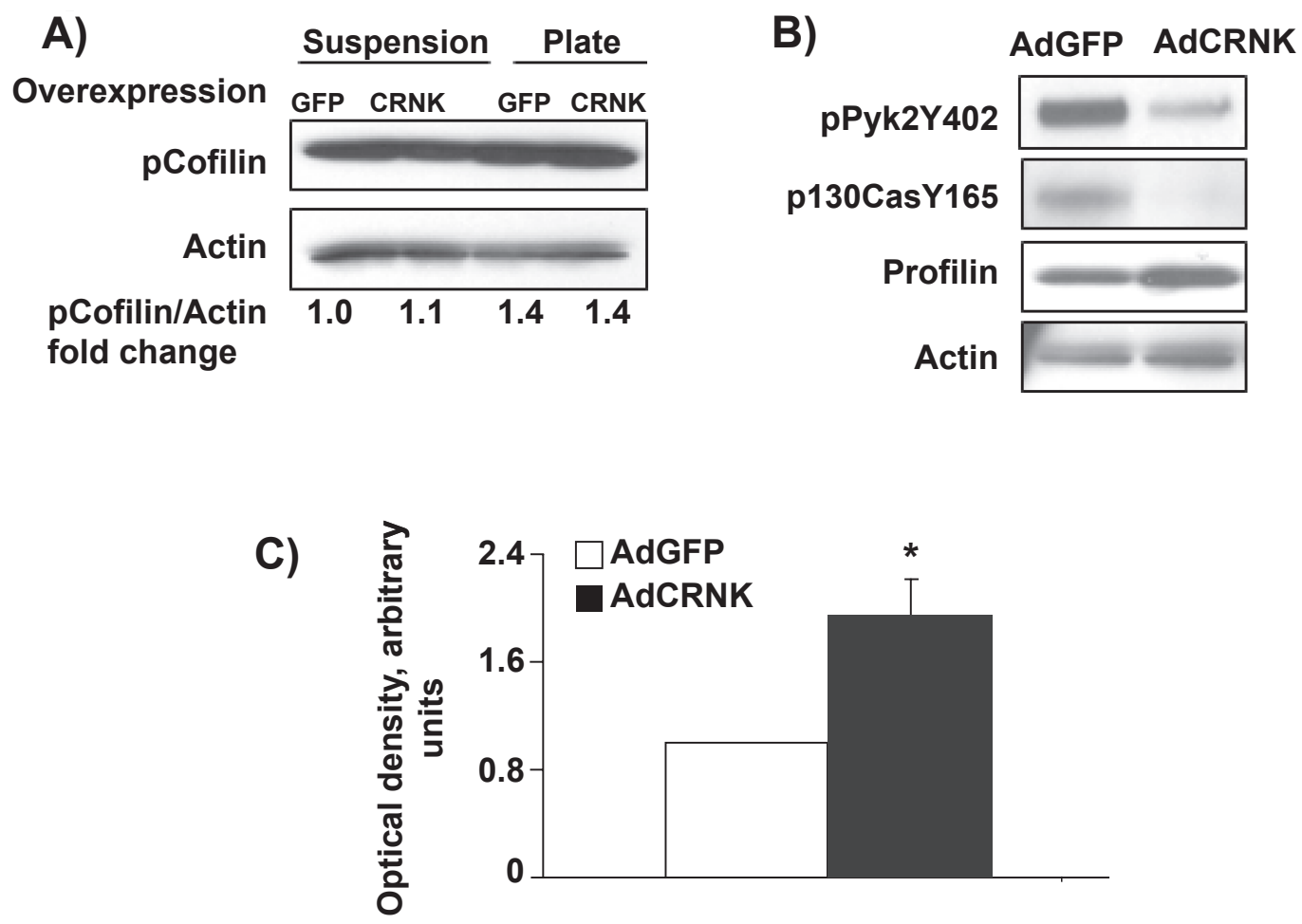

Figure 2 Inhibition of endogenous Pyk2 phosphorylation associated with increased profilin expression A) Cofilin phosphorylation does not change after endogenous Pyk2 activity inhibition with CRNK expression. Representative example of five experiments is shown. No significant difference in cofilin phosphorylation between GFP- and CRNK-expressing GMC were detected after one hour of spreading. B) Profilin protein level was increased after CRNK overexpression in GMC. Lysates from one hour re-plated AdGFP- and AdCRNK-infected human GMC were resolved by SDS-PAGE and immunoblotted either with anti-phosphorilated tyrosin 402 of Pyk2 (top panel), or with anti-profilin antibody (second panel from the top), or with anti-phosphorilated tyrosin I65 of pI30Cas (third panel from the top), or with anti-actin antibody (bottom panel) for loading control. Decreases in Pyk2 and pl30Cas phosphorylation after CRNK expression were associated with increase in profilin level. C) Results of four independent experiments analyzing profilin expression summarized graphically. Densitometry data normalized to AdGFP control and presented as mean \pm SEM.

Notes: *statistically significant difference $(p<0.05)$ compared to control. In average profiling expression was increased twice after CRNK expression.

Abbreviations: CRNK, calcium regulated nonkinase; GFP, green fluorescent protein; GMC, glomerular mesangial cell; Pyk2, proline-rich tyrosine kinase 2; SEM, standard error of mean.

actin in axons and growth cones of differentiated PC12 cells, ${ }^{29}$ suggesting RhoA activity importance for maintaining of intact cytoskeleton and corresponding Pyk2 translocation. Furthermore, RhoA-dependent regulation of the actin cytoskeleton selectively regulates smooth muscle cell differentiation marker gene expression by modulating serum response transcriptional factor (SRF) translocation to the nucleus and by altering SRFdependent transcription activity. ${ }^{16,30}$ These findings suggest that RhoA signaling may serve as a convergence point for the multiple signaling pathways that regulate cell type-specific differentiation. Additionally, post-transcriptional mechanisms may, under certain conditions, influence the level of differentiation marker proteins including profilin, as shown recently in serum-starved airway smooth muscle cells. Accumulation of SM22 and smooth muscle myosin heavy chain is regulated independently of mRNA level by a pathway involving phosphatidylinositide-3-kinase (PI3K), mammalian target of rapamycin (mTOR), and p70 S6 kinase..$^{31}$ Our finding of
Pyk2 activity correlation 1) with basal RhoA GTP-loading, 2 ) with ROCK activity and cell adhesion status, and 3 ) with profilin protein level, taken together with presented above line of evidences may explain that increased protein expression of profilin observed in our experiments by modulation Pyk2- and RhoA-dependent transcriptional mechanisms in GMC. Yet, further work needs to be done to support this hypothesis.

Alternatively, the increase in profilin protein can be explained by changes in protein stability during cell spreading. Some high dynamic cellular processes, including actin assembly and degradation, can be rapidly regulated by posttraslational mechanisms. Post-translational modifications by SUMO can increase FAK kinase activity. ${ }^{32}$ Pyk2 and CRNK have several sites suitable for SUMO modifications with unknown functional consequences (SUMOplot Prediction; Abgent, San Diego, CA). Yeast profilin homolog, Cdc3, was shown to be modified by SUMO and this modification was important for proper actin dynamic during budding of 


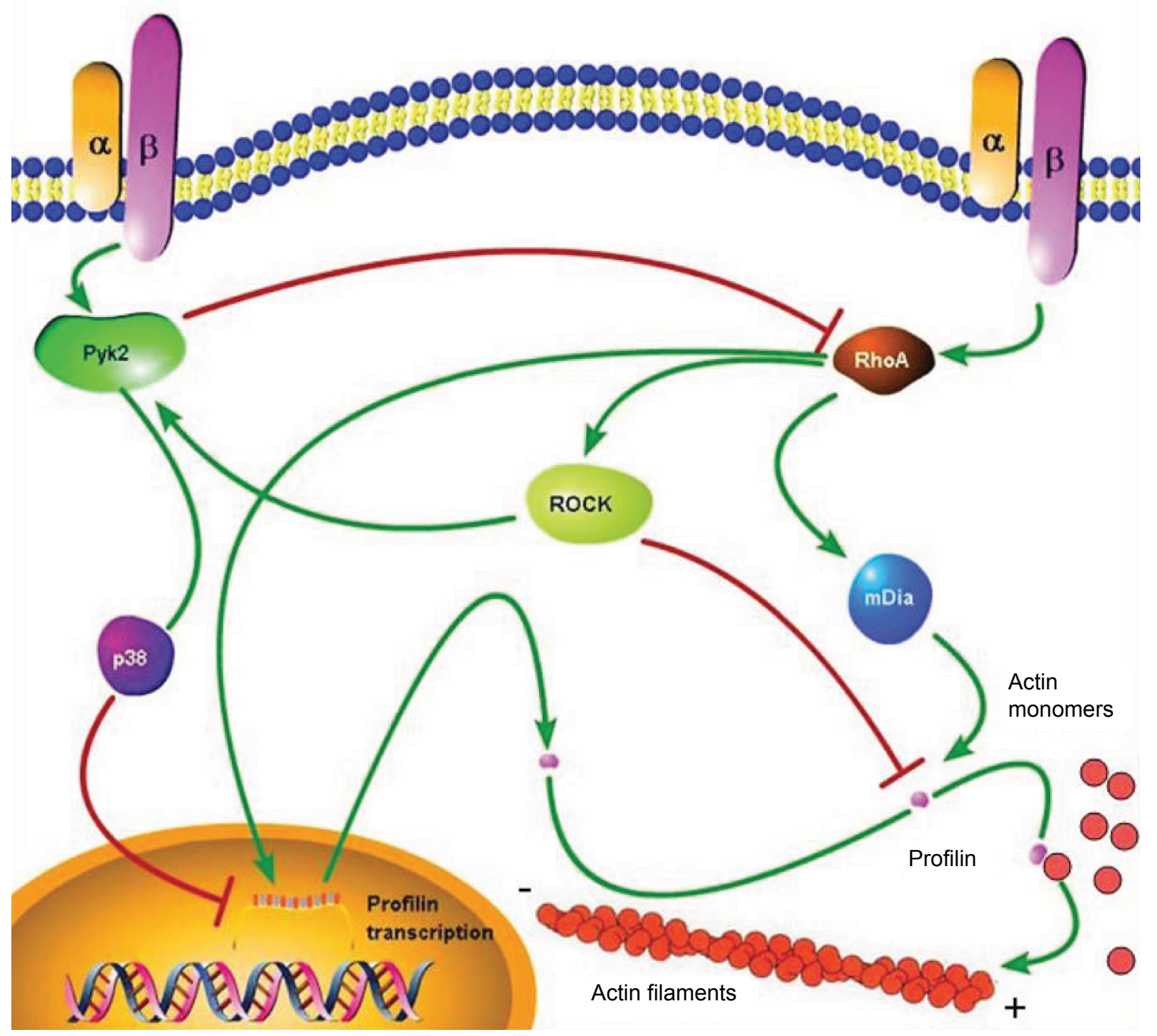

Figure 3 The proposed model of Pyk2 and RhoA signaling interactions resulting in changes in profilin expression. Heterodimeric integrin receptors could potentially activate Rho via signals originating from cytoplasmic domain of beta-integrin subunit at focal adhesions, and thus the mechanisms activating the Rho and Pyk2 pathways would partially overlap. In the present study we report that in human GMC de-novo adhesion to substrate was significantly decreased after Pyk2 activity inhibition and was further diminished after treatment with ROCK kinase inhibitor, suggesting cross talk between Pyk2 and RhoA signaling. RhoA can stimulate profilin activity toward actin filament assembling via $\mathrm{mDia}$. At the same time, RhoA-dependent activation of ROCK may result in inhibition of profilin activity and stimulation of Pyk2 activity. Effect upon Pyk2 activity could be mediated by ROCK-triggered changes in actin cytoskeleton. Furthermore, RhoA-dependent regulation of the actin cytoskeleton may promote profilin gene expression. Pyk2 activation can be important for focal adhesion sites formation. According to our data collected from human glomerular mesangial cells, Pyk2 also can diminish RhoA activity status, activate p38 kinase, and may lead to inhibitory effect on profilin expression. Decrease of Pyk2 activation with CRNK overexpression (see text for more details) caused overall balance shifted toward increased profilin expression. RhoA-ROCK-LIMK-Cofilin pathway is not shown. Inhibitory interactions are shown by red lines with bars. Stimulatory interactions are shown by green lines with arrows.

Abbreviations: CRNK, calcium regulated nonkinase; GFP, green fluorescent protein; GMC, glomerular mesangial cell; Pyk2, proline-rich tyrosine kinase 2; ROCK, Rhoassociated kinase; SEM, standard error of mean.

daughter cell. ${ }^{33}$ So we cannot exclude the possibility that post-translational modifications of profilin or upstream signaling pathways can be involved into stabilization of protein in CRNK expressing GMC population. Studies of SUMOylation or ubiquitination of Pyk2, CRNK and profilin would help to clarify this issue. A Rap1-GTP-interacting adaptor molecule (RIAM) is interacting with profilin, linking
Rap1 and, correspondingly, Pyk2 to integrin activation and actin dynamics. ${ }^{34}$

The ratio of globular to filamentous actin in spreading CRNK-expressing cells compared with control cells was decreased. This observation suggests that level of Pyk2 activity can affect actin polymerization and shift the local balance between assembly and disassembly of actin towards 
cortical F-actin accumulation. Plausible explanation may lay in formation of focal adhesions, which connect cells and the extracellular matrix. Sawada and colleagues ${ }^{35}$ reported that the mechanical stretching of cells induced p130Cas stretching, an adaptor protein at focal adhesions, to undergo a conformational change. This change promotes phosphorylation of p130Cas by Src family kinases and the transduction of integrin-mediated signaling. Correspondently, decreased p130Cas phosphorylation after inhibited Pyk2 activity may result in reduced number of focal adhesions formed or in impairment of their maturity (ie, increase in size). Therefore, the reduced number of or impaired focal adhesions affected F-actin formation. It is plausible that obtained data can be explained if in GFP-expressing GMC filamentous actin formation during cell spreading characterized by multiple branching of actin assembling network, and in the CRNK population it would be fewer origins of actin monomers polymerization resulting in longer filaments. Indeed, with more barber $(+)$ ends and shorter filaments in actin network presented, the addition of cytochalasin $\mathrm{D}$ will result in relative decrease of filamentous actin compared to network with less globular actin assembling sites and longer filaments (Figure 1C). Additionally, stabilization of the filamentous actin with phalloidin suppose result in similar or less relative amount of F-actin detected in case of longer but fewer filaments compared to more branched network. Molecular mechanisms regulating actin network architecture may include CRNK interference 1) with Ena/VASP actin branching activity via interaction with Arp2/3; and/or 2) with Ena/ VASP activity to enhance actin filament elongation via the recruitment of profilin-actin complexes to sites of active actin remodelling such as the tips of spreading lamellipodia. ${ }^{36}$

The physiological role of GMC in glomerulus is to provide structural support for and regulate blood flow of the glomerular capillaries by the contractile activity toward fenestrated endothelium and glomerular basement membrane. GMC contraction decreases the surface area of the basement membrane therefore directly affecting glomerular filtration rate. The connection between Pyk 2 and profilin might have functional importance for GMC especially during disease-associated changes. In cultured rat mesangial cells, expression of profilin mRNA and protein was upregulated by basic fibroblast growth factor (bFGF) but not by plateletderived growth factor or transforming growth factor-beta (TGF-beta). Similarly to profilin, Pyk2 is activated by bFGF stimulation resulting in $\mathrm{p} 38$ and JNK signaling induction. ${ }^{37,38}$ Therefore, Pyk2 and profilin may be united in hypothetical signaling cascade. However, TGF-beta specifically induces profilin expression in the myofibroblast, ${ }^{39}$ suggesting cell-specific transcription regulation by different growth factors. Interesting that Pyk2 was shown to be involved into TGF-beta-induced signaling. Specifically, TGF-beta induced the phosphorylation of focal adhesion kinase Pyk2, and this effect was markedly increased in the presence of immobilized fibronectin, suggesting a collaborative role for fibronectinspecific integrins. ${ }^{40}$ Yet, it remains to be established whether fibronectin is indeed an important factor in Pyk2-mediated signaling in mesangial cells.

In summary, we have demonstrated that in human GMC de novo adhesion to substrate was significantly decreased after Pyk2 activity inhibition and was further diminished after treatment with ROCK inhibitor. Adenovirus-mediated CRNK expression was associated with increased formation of filamentous actin and a corresponding decrease in globular to filamentous actin ratio during cell spreading when compared with control cells. Profilin, an important activator of actin polymerization, expression was enhanced and this increase was associated with decrease in Pyk2 and p130Cas phosphorylations in CRNK-expressing GMC compared to control cells. Our data suggest Pyk2 signaling cascade may control the filamentous actin formation during cell spreading via regulation of profilin expression.

\section{Disclosure}

The authors report no conflicts of interest in this work. This study was supported by NIH research grants RO1 DK 41684 and RO1 HL 22563 to A. Sorokin and by AHA Postdoctoral Fellowship 0525801Z to V. Rufanova (Petrukhina).

\section{References}

1. Avraham S, London R, Fu Y, et al. Identification and characterization of a novel related adhesion focal tyrosine kinase (RAFTK) from megakaryocytes and brain. J Biol Chem. 1995;270:27742-27751.

2. Martinez MC, Randriamboavonjy V, Ohlmann P, et al. Involvement of protein kinase $\mathrm{C}$, tyrosine kinases, and Rho kinase in $\mathrm{Ca}(2+)$ handling of human small arteries. Am J Physiol Heart Circ Physiol. 2000;279: H1228-H1238.

3. Lev S, Moreno H, Martinez R, et al. Protein tyrosine kinase PYK2 involved in $\mathrm{Ca}(2+)$-induced regulation of ion channel and MAP kinase functions. Nature. 1995;376:737-745.

4. Tolloczko B, Tao FC, Zacour ME, Martin JG. Tyrosine kinase-dependent calcium signaling in airway smooth muscle cells. Am J Physiol Lung Cell Mol Physiol. 2000;278:L1138-L1145.

5. Watts SW. 5-Hydroxytryptamine-induced potentiation of endothelin-1and norepinephrine-induced contraction is mitogen-activated protein kinase pathway dependent. Hypertension. 2000;35:244-248.

6. Zubkov AY, Rollins KS, Parent AD, Zhang J, Bryan RM Jr. Mechanism of endothelin-1-induced contraction in rabbit basilar artery. Stroke. 2000;31:526-533.

7. Sorokin A, Kozlowski P, Graves L, Philip A. Protein-tyrosine kinase Pyk2 mediates endothelin-induced p38 MAPK activation in glomerular mesangial cells. J Biol Chem. 2001;276:21521-21528. 
8. Sorokin A, Kohan DE. Physiology and pathology of endothelin-1 in renal mesangium. Am J Physiol Renal Physiol. 2003;285:F579-F589.

9. Rufanova VA, Alexanian A, Wakatsuki T, Lerner A, Sorokin A. Pyk2 mediates endothelin-1 signaling via p130Cas/BCAR3 cascade and regulates human glomerular mesangial cell adhesion and spreading. J Cell Physiol. 2009;219:45-56.

10. Ivankovic-Dikic I, Gronroos E, Blaukat A, Barth BU, Dikic I. Pyk2 and FAK regulate neurite outgrowth induced by growth factors and integrins. Nat Cell Biol. 2000;2:574-581.

11. Clark EA, Brugge JS. Integrins and signal transduction pathways: the road taken. Science. 1995;268:233-239.

12. Hellstrand P, Albinsson S. Stretch-dependent growth and differentiation in vascular smooth muscle: role of the actin cytoskeleton. Can J Physiol Pharmacol. 2005;83:869-875.

13. Goode BL, Eck MJ. Mechanism and function of formins in the control of actin assembly. Annu Rev Biochem. 2007;76:593-627.

14. Numaguchi K, Eguchi S, Yamakawa T, Motley ED, Inagami T. Mechanotransduction of rat aortic vascular smooth muscle cells requires RhoA and intact actin filaments. Circ Res. 1999;85:5-11.

15. Sotiropoulos A, Gineitis D, Copeland J, Treisman R. Signal-regulated activation of serum response factor is mediated by changes in actin dynamics. Cell. 1999;98:159-169.

16. Mack CP, Somlyo AV, Hautmann M, Somlyo AP, Owens GK. Smooth muscle differentiation marker gene expression is regulated by RhoAmediated actin polymerization. J Biol Chem. 2001;276:341-347.

17. Birbach A. Profilin, a multi-modal regulator of neuronal plasticity. Bioessays. 2008;30:994-1002.

18. Shao J, Welch WJ, Diprospero NA, Diamond MI. Phosphorylation of profilin by ROCK1 regulates polyglutamine aggregation. Mol Cell Biol. 2008;28:5196-5208.

19. Hall A. Rho GTPases and the actin cytoskeleton. Science. 1998;279: 509-514.

20. Sraer JD, Delarue F, Hagege J, et al. Stable cell lines of T-SV40 immortalized human glomerular mesangial cells. Kidney Int. 1996;49:267-270.

21. Rufanova VA, Sorokin A. CrkII associates with BCAR3 in response to endothelin-1 in human glomerular mesangial cells. Exp Biol Med (Maywood). 2006;231:752-756.

22. Ostergaard HL, Lysechko TL. Focal adhesion kinase-related protein tyrosine kinase Pyk2 in T-cell activation and function. Immunol Res. 2005;31:267-282.

23. Loeser RF, Forsyth CB, Samarel AM, Im HJ. Fibronectin fragment activation of proline-rich tyrosine kinase PYK2 mediates integrin signals regulating collagenase- 3 expression by human chondrocytes through a protein kinase C-dependent pathway. J Biol Chem. 2003;278:24577-24585.

24. Gupta S, Clarkson MR, Duggan J, Brady HR. Connective tissue growth factor: potential role in glomerulosclerosis and tubulointerstitial fibrosis. Kidney Int. 2000;58:1389-1399.

25. Ruiz-Torres MP, Lopez-Ongil S, Griera M, Diez-Marques ML, Rodriguez-Puyol M, Rodriguez-Puyol D. The accumulation of extracellular matrix in the kidney: consequences on cellular function. J Nephrol. 2005; $18: 334-340$
26. Defilippi P, Olivo C, Venturino M, Dolce L, Silengo L, Tarone G. Actin cytoskeleton organization in response to integrin-mediated adhesion. Microsc Res Tech. 1999;47:67-78.

27. Hopkins AM, Pineda AA, Winfree LM, Brown GT, Laukoetter MG, Nusrat A. Organized migration of epithelial cells requires control of adhesion and protrusion through Rho kinase effectors. Am J Physiol Gastrointest Liver Physiol. 2007;292:G806-G817.

28. Lee MS, Kim YB, Lee SY, et al. Integrin signaling and cell spreading mediated by phorbol 12-myristate 13-acetate treatment. J Cell Biochem. 2006;99:88-95.

29. Haglund K, Ivankovic-Dikic I, Shimokawa N, Kruh GD, Dikic I. Recruitment of Pyk2 and $\mathrm{Cbl}$ to lipid rafts mediates signals important for actin reorganization in growing neurites. $J$ Cell Sci. 2004;117:2557-2568.

30. Liu HW, Halayko AJ, Fernandes DJ, et al. The RhoA/Rho kinase pathway regulates nuclear localization of serum response factor. $\mathrm{Am} J$ Respir Cell Mol Biol. 2003;29:39-47.

31. Halayko AJ, Kartha S, Stelmack GL, et al. Phophatidylinositol-3 kinase/mammalian target of rapamycin/p70S6K regulates contractile protein accumulation in airway myocyte differentiation. Am J Respir Cell Mol Biol. 2004;31:266-275.

32. Kadare G, Toutant M, Formstecher E, et al. PIAS1-mediated sumoylation of focal adhesion kinase activates its autophosphorylation. J Biol Chem. 2003;278:47434-47440.

33. Takahashi Y, Iwase M, Konishi M, Tanaka M, Toh-e A, Kikuchi Y Smt3, a SUMO-1 homolog, is conjugated to Cdc3, a component of septin rings at the mother-bud neck in budding yeast. Biochem Biophys Res Commun. 1999;259:582-587.

34. Lafuente EM, van Puijenbroek AA, Krause M, et al. RIAM, an Ena/ VASP and Profilin ligand, interacts with Rap1-GTP and mediates Rap1-induced adhesion. Dev Cell. 2004;7:585-595.

35. Sawada Y, Tamada M, Dubin-Thaler BJ, et al. Force sensing by mechanical extension of the Src family kinase substrate p130Cas. Cell. 2006;127:1015-1026.

36. Sechi AS, Wehland J. ENA/VASP proteins: multifunctional regulators of actin cytoskeleton dynamics. Front Biosci. 2004;9:1294-1310.

37. Manso AM, Kang SM, Plotnikov SV, et al. Cardiac fibroblasts require focal adhesion kinase for normal proliferation and migration. Am J Physiol Heart Circ Physiol. 2009;296:H627-H638.

38. Liu ZY, Ganju RK, Wang JF, et al. Characterization of signal transduction pathways in human bone marrow endothelial cells. Blood. 1997;90:2253-2259.

39. Malmstrom J, Lindberg H, Lindberg C, et al. Transforming growth factor-beta 1 specifically induce proteins involved in the myofibroblast contractile apparatus. Mol Cell Proteomics. 2004;3:466-477.

40. Brill A, Franitza S, Lider O, Hershkoviz R. Regulation of T-cell interaction with fibronectin by transforming growth factor-beta is associated with altered Pyk2 phosphorylation. Immunology. 2001;104:149-156.
Cell Health and Cytoskeleton

\section{Publish your work in this journal}

Cell Health and Cytoskeleton is an international, peer-reviewed open access journal focusing on all aspects of cell structure and function contributing to normal physiology and cell health and exploring the pathogenesis of cell dysfunction leading to adverse conditions and disease in the organism. The journal welcomes papers covering original research,

\section{Dovepress}

basic science, reviews and evaluations, guidelines, expert opinion and commentary, case reports and extended reports. The manuscript management system is completely online and includes a very quick and fair peerreview system, which is all easy to use. Visit http://www.dovepress.com/ testimonials.php to read real quotes from published authors. 